\title{
Human-Animal Relationships: The Use of Species-Typical Food Calls and Chimpanzee (Pan troglodytes) Names: Welfare-Oriented Tools to Manage Sanctuary Chimpanzees
}

\author{
Leilani Case $^{1 *}$, Akie Yanagi ${ }^{1}$, Erin Loeser ${ }^{1}$, and Amy Fultz ${ }^{1}$
}

${ }^{1}$ Chimp Haven

*Corresponding author (Email: lcase@ chimphaven.org)

Citation - Case, L., Yanagi, A., Loeser, E., \& Fultz, A. (2015). Human-animal relationships: The use of speciestypical food calls and chimpanzee (Pan troglodytes) names: Welfare-oriented tools to manage sanctuary chimpanzees. Animal Behavior and Cognition, 2(3), 254-266. doi: 10.12966/abc.08.05.2015

\begin{abstract}
In captivity, positive human-animal interactions are often part of daily management procedures, which can enhance the welfare of animals and help caregivers with routine husbandry tasks. For example, the provisioning of food (produce or enrichment) can be an effective tool to reward animals for positive behavior. Another option is to use species-typical behavior to request an action on the part of the animal. In both captive and wild settings, chimpanzees (Pan troglodytes) use a variety of food vocalizations to announce the presence of different food sources. In this study, we examined how species-typical vocalizations (chimpanzee-based food calls, CBFCs) compared to human vocalizations (name-calling and encouragement, NCE) in relation to the shifting behavior of sanctuary chimpanzees at Chimp Haven, Inc., during routine husbandry tasks. Both CBFCs and NCE were associated with the provisioning of enrichment or produce, and observations were balanced for time of day. We found that CBFCs were slightly more effective in shifting of the chimpanzees when it was used along with the provisioning of enrichment $(n$ $=22, p=0.017)$. We also found that the chimpanzees were more likely to shift in the morning rather than in the afternoon $(n=34, p=0.012)$. These results suggest that predictable schedules and the use of positive communication, whether it is in the form of a species-typical food call or the calling of a chimpanzee's name and general positive encouragement, assisted in the daily management of chimpanzees housed in a sanctuary setting.
\end{abstract}

Keywords - Food call, Sanctuary, Chimpanzee, Welfare, Management, Species typical behavior

Determining the welfare of animals in captivity can be difficult due to variations in the ability of individual animals to cope with the stress and challenges of daily life, as well as differences in the methods employed to assess welfare (Dawkins, 1998, 2003). One way of measuring welfare is through behavioral assessments of the interaction between human caregivers and their animal charges (Chelluri, Ross, \& Wagner, 2013; Dawkins, 1998, 2003; Hosey \& Melfi, 2012). However, according to Hosey and Melfi (2012), most of the research on these human-animal relationships (HARs) involved companion animals (161 articles), while relatively few studies were conducted on laboratory animals or zoo animals $(18,22)$. At this time, the authors are unaware of any articles that have been published that specifically focus on sanctuary animals (Hosey \& Melfi, 2012).

The relationship may vary based on the captive setting. In the majority of zoos, the public, veterinary staff, and caregivers are part of the daily lives of the animals (Carlstead, 2009; Chelluri et al. 2013; Hosey \& Melfi, 2012). With laboratory animals, veterinary staff, caregivers, and researchers are the most common visitors (Bayne, 2002). Animal sanctuaries usually employ caregivers and veterinary staff while limiting the exposure of the animals to researchers or the public (Brent, 2007). 
In zoos and laboratories, the ability of caregivers to interact physically with the animals varies by species, facility, and task (Bloomsmith, Stone, \& Laule, 1998; Carlstead, 2009; Perlman et al., 2012). In general, for large species, including great apes, caregivers do not enter an enclosure with the animal (Dorsey, 2010). Instead, animals must voluntarily move from one part of their enclosure to another for cleaning, maintenance, health checks, and other general husbandry tasks (Perlman et al., 2012).

There is evidence that, when there is a positive and reciprocal HAR, animals have reduced stress and cortisol levels, greater reproduction and production potential, and more efficiently cooperate in daily husbandry tasks (Bayne, 2002; Bloomsmith et al., 1998; Hemsworth, 2007; Hosey, 2008; Hosey \& Melfi, 2012; Jensvold, 2008; Perlman et al., 2012). Animals can be negatively impacted by subtle behaviors such as the volume and tone of human voice and the noise of keys jingling (Carlstead, 2009; Gibson, Scavelli, Udell, \& Udell, 2014; Hemsworth, 2003).

The most common interaction between captive animals and caregivers is during routine husbandry procedures, which include feeding and moving or shifting animals from one area to another for cleaning purposes and administering medications. Usually, these routine husbandry procedures are at least mildly stressful for the animals (Bassett, Buchanan-Smith, McKinley, \& Smith, 2003; Reinhardt, 2004; Saibaba, Sales, Stodulski, \& Hau, 1996; Waitt, Buchanan-Smith, \& Morris, 2002). For example, self-scratching, a behavior related to stress, is higher during regular husbandry procedures for the common marmoset (Callithrix jacchus) (Bassett et al., 2003). At Lincoln Park Zoo, stress related behaviors such as selfscratching, self-directed behavior and aggression increased during the period of time chimpanzees were shifted into a smaller off-view holding area for daily maintenance of their exhibit (Ross, Wagner, Schapiro, \& Hau, 2010). Similarly, aggression, activity, and locomotion increased slightly while social behaviors decreased in chimpanzees during space-restricted periods (Aureli \& de Waal, 1997; Nieuwenhuijsen \& de Waal, 1982). In addition, parturition in chimpanzees is typically delayed until the weekend nights when caregiver presence is lowest (Alford, Nash, Fritz, \& Bowen, 1992). Lambeth et al. (1997) found wounding rates to be highest during the week (average of 70.6 episodes of wounding), when more caregivers are present and most veterinary procedures, such as sedations for physicals, occur, compared to the weekend (average of 19.5 wounding episodes).

At the same time, regular husbandry tasks can provide an opportunity for positive interaction, enrichment, and trust building between the animals and their caregivers (Claxton, 2011). Shifting, or moving animals from one area to another, is frequently reinforced by the provisioning of food items. As movement to a different location is a voluntary choice that can be refused, animal caregivers typically seek humane methods to encourage this movement. Positive reinforcement training for shifting animals into different areas, especially during stressful veterinary or husbandry procedures, helps maintain or improve the HAR and increase efficiency during daily husbandry (Lambeth, Hau, Perlman, Martino, \& Schapiro, 2006; Perlman et al., 2012).

Although various factors, such as personality or the upbringing of the animal, may also have an impact on the HAR (Bayne, Dexter, \& Strange, 1993), there is evidence of a relationship between the HAR and the welfare of many captive animals (Carlstead, 2009; Chelluri et al., 2013; Hosey \& Melfi, 2012). Studies on chimpanzees have found that positive reinforcement training is much more effective and efficient for research and husbandry purposes in the laboratory, as compared to negative or neutral interactions (Bloomsmith et al., 1998; Kessel-Davenport \& Gutierrez, 1994). Positive reinforcement training has also helped reduce abnormal behaviors while increasing prosocial behaviors in a zoo setting among chimpanzees (Pomerantz \& Terkel, 2009). However, Chelluri et al. (2013) found positive informal interactions to reduce abnormal behavior but increase agonistic behavior among zoo-housed chimpanzees and gorillas (Gorilla gorilla gorilla). The use of species-typical behavior can increase affiliative relationships between caregivers and animals (e.g., chimpanzees: Jensvold, 2008), as well as affiliative behaviors between conspecifics, such as grooming (e.g., rhesus macaques, Macaca mulatta, Bayne et al., 1993), while decreasing aggression toward caregivers.

Effective communication between humans and animals is an important factor in fostering positive HARs, as most species communicate in different ways. Chimpanzees, for example, have a complex system of vocalizations, some of which lead to a behavioral response on the part of the listener. In studies of wild 
chimpanzees, particular types of vocalizations, known as food calls, communicate the presence of food and often attract other chimpanzees to the food source (Goodall, 1986). As chimpanzees approach and feed at a large and valuable resource, a medley of calls, including food grunts, food "aaa" calls, pant-hoots, food barking, and screams, can be heard from all members of the group (Goodall, 1986; Mitani, 1994). Chimpanzees use specific types of food calls in order to share the quality and quantity of foods with group members (Brosnan \& de Waal, 2014; Slocombe et al., 2010; Slocombe \& Zuberbühler, 2006). The loudest food calls, including pant-hoots, loud "aaa" calls, and barks, are especially important to convey information about large and valuable food sources to distant members of the group (Brosnan \& de Waal, 2014; Goodall, 1986; Mitani, 1994; Slocombe et al., 2010; Slocombe \& Zuberbühler, 2006) as it is common for chimpanzees to be at considerable distances while feeding during the day due to their fission-fusion social network (Goodall, 1986). Captive chimpanzees also produce food calls in the presence of food and produce more calls with greater quantities of food (Brosnan \& de Waal, 2002).

Some studies have focused on captive chimpanzees communicating with human caregivers in relation to the acquisition of food (e.g., Hopkins, Taglialatela, \& Leavens, 2007; Hostetter, Cantero, \& Hopkins, 2001). Hostetter et al. (2001) found that chimpanzees understand the attentive state of humans in relation to food (i.e., if they were oriented to see the food or not) and will produce calls appropriate to the situation. The chimpanzees produced food calls when the human was not looking at the food but used hand gestures when the human was oriented toward the food. In another study, $71 \%$ of the chimpanzee subjects produced attention-getting calls when both the human and food were present compared to $29 \%$ for human only and $8 \%$ for food only (Hopkins et al., 2007). However, few studies have focused on the role of communication by caregivers to chimpanzees using species-typical behavior. Two exceptions are the studies conducted by Jensvold (2008) and Itakura et al. (1999). Jensvold (2008) attempted to communicate with the chimpanzee by having caregivers use more chimpanzee-appropriate gestures, resulting in decreased aggressive action aimed at caregivers and increased positive interactions such as grooming and play. Itakura et al. (1999) found that most chimpanzees in their laboratory were skilled at locating the food with a food call, regardless of informant (chimpanzee or human) or type of vocal cue (species-typical call or nonsense two-syllable words), but were not as successful in acquiring food when relying on their caregivers' gaze. Whether such caregiver-animal communication, using species-typical vocalizations, has similar effects in non-laboratory settings, such as sanctuaries, is unknown.

Here we provide the first example of using a species-typical vocalization for husbandry purposes in a sanctuary setting. Specifically, we examine the relationship of shifting chimpanzees through positive verbal communication with caregivers to enhance their management. Given the importance of both the role of vocalizations to indicate the presence and quantity of food to chimpanzees and positive human-animal interactions, the specific goal of this study was to determine whether using a chimpanzee-based food call (CBFC) or calling the chimpanzees with encouraging words and their names (i.e., name-calling with encouragement (NCE)) would be more efficient in shifting chimpanzees for regular husbandry procedures, including cleaning and produce enrichment provisioning. We hypothesized that a caregiver distributing produce or enrichment, while producing CBFCs, would facilitate shifting more often than NCE due to the more species-typical behavior employed by the caregiver. Further, we hypothesized that the afternoon would be more conducive to shifting behavior due to the fact that chimpanzees are more likely to shift when stress related to husbandry is lowest. Although the effects of time of day may be important to consider for the management of chimpanzees, studies examining such effects are virtually non-existent to date. Specifically, we examined the following predictions: (a) chimpanzees were more likely to shift to the outdoor enclosure when we used CBFCs than when we used NCE and (b) chimpanzees were more likely to shift to the outdoor enclosure during afternoon hours, when veterinary procedures, husbandry presence, and the need for shifting and cleaning are lowest. 


\section{Method}

\section{The Study Site}

The study took place at Chimp Haven, Inc., located in northwest Louisiana. Chimp Haven is a sanctuary designed to provide humane care to more than two hundred retired biomedical-research, pet, and performer chimpanzees through complex environments and enriched permanent housing. Four social groups housed at Chimp Haven were the subjects of this study (details provided below).

\section{Enclosures}

Each group had access to three to five indoor areas called bedrooms (each $192 \mathrm{ft}^{2} / 17.8 \mathrm{~m}^{2}$ ), which are ventilated at all times and heated during cold weather. The bedrooms are connected to each other and to outdoor enclosures through a series of doors and chutes.

The four groups of chimpanzees were rotated among forested outdoor habitats, open air corrals, and an outdoor yard such that one group occupied one of the primary outdoor areas (habitat, corral, or yard) and associated indoor bedrooms. Habitats measure 217,800 ft ${ }^{2}\left(20.16 \mathrm{~m}^{2}\right)$ and 174, $240 \mathrm{ft}^{2}\left(16.17 \mathrm{~m}^{2}\right)$; both are natural secondary forest with natural ground covering. The areas are contained on two sides by poured concrete walls 17 to $20 \mathrm{ft}$ ( 5.2 to $6.1 \mathrm{~m}$ ) high with mesh windows at eye level. The third side of the habitats are bound by a water moat, which measures approximately $60 \mathrm{ft}(18.3 \mathrm{~m}$ ) across (see Fultz, Brent, Breaux, $\&$ Grand, 2013 for a detailed description). When a chimpanzee group is rotated out of the habitat, they have access to one of two $5,776 \mathrm{ft}^{2}\left(536.6 \mathrm{~m}^{2}\right)$ open air corrals contained by a poured concrete wall $17 \mathrm{ft}(5.2 \mathrm{~m})$ high with mesh windows at eye level or a $3,700 \mathrm{ft}^{2}\left(343.74 \mathrm{~m}^{2}\right)$ yard. Both the corrals and the yard have natural-grass ground covering and wooden climbing structures. During both stages of the study, the chimpanzee groups occupied one specific location for the majority of the study period, but on rare occasions the chimpanzees were temporarily rotated between areas due to management concerns such as sedations, an injured or ill individual, or introductions.

The chimpanzees have access to both indoor and outdoor areas at all times, day and night, except during sanitation of the enclosures. This is unlike a zoo setting where chimpanzees are typically locked inside at night and let into outdoor areas in the morning (Coe, Fulk, \& Brent, 2001, Ross et al., 2010). Cleaning of indoor bedrooms is completed daily in the morning and the chimpanzees are shifted outside to do so; they are locked outside for a short period during this time. Cleaning of outdoor enclosures occurs every two weeks and the chimpanzees are locked inside during this time. Cleaning is one of the few times that the chimpanzees at Chimp Haven are locked inside (other times might include during inclement weather or when repairs to the area are necessary). Chimpanzees were always shifted from smaller indoor areas to larger outdoor areas during each stage of the study, regardless of time of day.

\section{Diet}

The chimpanzees are fed a commercially available primate diet twice per day and fruits and vegetables twice per day. Morning produce consists of two fruits, a vegetable, a starch, and greens; chimpanzees receive a vegetable in the afternoon. The morning produce is used to shift animals to the outdoor enclosure to allow inside areas to be cleaned and sanitized. Forage mix (including various seeds, nuts, and cereals) is scattered twice a week. Enrichment, which includes both food and non-food items, is provided daily; food-based enrichment is generally provided four times a week. During the study, the chimpanzees received all of their daily produce and enrichment regardless of condition of the study. The study was conducted in two stages due to questions that arose during the first stage of the study. 


\section{Stage 1}

Stage 1 took place from September 2009 to February 2009.

Subjects. Fifty-six chimpanzees (Male $=26$, Female $=30$ ) in four separate social groups with an age range of 1 to 50 years old $(M=32.39)$ were part of the Stage 1 study. The chimpanzees were housed in multi-male, multi-female social groups ranging in size from 11 to 17 individuals $(M=14.25)$. The groups were observed during nine trials for each of four conditions, for a total of 2,016 observations.

Procedure. To determine the role of the type of vocalization, we examined when a caregiver produced a CBFC versus NCE; one call type was used in each condition. During the CBFC conditions, a caregiver distributed either produce (CBFCP) or enrichment (CBFCE) while she/he made CBFC vocalizations. The human caregiver mimicked the loudest calls, food barks, as described by Goodall (1986). During the NCE conditions, produce (NCEP) or enrichment (NCEE) was distributed while the caregiver provisioning the items called the chimpanzees by name and used encouraging statements such as "Come on outside," "Excellent, Henry," and "Good job, everybody." Both daily produce and food enrichment were distributed into the chimpanzees' outdoor enclosures from a rooftop platform. Enrichment items were all food based and were distributed in the afternoon by an enrichment technician. Produce was predominantly distributed by other caregivers who generally performed standard animal husbandry routines in the morning. Both CBFCs and NCE were produced periodically for up to five minutes, at which point data recording began. Chimpanzees were recorded as having shifted when they entered the outdoor enclosure and were visible to the observer for the next five minutes; if they did not come to the outdoor enclosure in this timeframe, we recorded this as not shifting. Food items were given to the chimpanzees when they arrived in the outdoor enclosure with each chimpanzee receiving at least one item. After data collection was complete, all chimpanzees received either the produce or enrichment regardless of their movement.

\section{Stage 2}

After the conclusion of Stage 1, we considered enrichment in the afternoon, produce in the morning, and the natural variations of vocalizations between caregivers to be possibly confounding variables. To control for this, we completed Stage 2 of the study. Stage 2 took place from December 2011 to July 2012.

Subjects. Subjects were 49 chimpanzees (Male $=21$, Female $=28$ ) in four separate social groups with 9 to 15 individuals $(M=12.5)$ ranging in age from 4 to 59 years old $(M=34.10)$. Group composition differed between Stage 1 and Stage 2 due to introductions and transfers to new groups, as well as deaths of group members due to typical disease processes associated with aging in chimpanzees. The groups were observed during ten trials for each of four conditions, for a total of 1,960 observations.

Procedure. During Stage 2 an enrichment technician was the only individual who distributed the daily produce, and made the two types of vocalizations (CBFC and NCE). Enrichment items were excluded in this stage of the study. As most husbandry procedures, which are potentially stressful, occurred in the morning hours, we examined differences between morning (before 12 PM CST: AM) and afternoon (after 12:10 PM CST: PM). Thus, the four conditions tested in Stage 2 were: CBFCAM, CBFCPM, NCEAM, and NCEPM.

\section{Data Analysis}

All tests were conducted with two-tailed tests, using IBM SPSS version 22. For prediction (a), in order to compare the chimpanzees' shifting behavior between two vocalization types (CBFC versus NCE) without the effect of other variables (i.e., food items for Stage 1 and time of day for Stage 2), we first calculated the proportion of shifts to the outdoor enclosure per individual chimpanzee for each vocalization type, combining two specific conditions (Stage 1: CBFCP + CBFCE for total CBFC versus NCEP + NCEE for total NCE; Stage 2: CBFCAM + CBFCPM for total CBFC versus NCEAM + NCEPM for total NCE), 
and performed a Wilcoxon matched-pairs signed-rank test. For Stage 2, we additionally examined the general tendency of shifting based on time of day (regardless of the call types), using the same test, by combining CBFCAM and NCEAM versus CBFCPM and NCEPM.

For the analyses involving more precise conditions, similar proportions of shifts were calculated for the four different conditions for each stage, respectively (Stage 1: CBFCP, CBFCE, NCEP, and NCEE; Stage 2: CBFCAM, CBFCPM, NCEAM, and NCEPM). For both stages, due to the non-normally distributed data, we used the Friedman non-parametric analysis of variance (Friedman ANOVA) to compare the proportions of shifts across the four conditions. A probability of $p \leq 0.05$ was considered significant and $0.05<p \leq 0.1$ was considered to be a non-significant tendency.

As there were significant differences across the four conditions based on Friedman ANOVA for both stages, we followed up on the findings by post hoc Wilcoxon matched-pairs signed-rank tests. Specifically, for Stage 1, we compared two sets of combinations of the conditions to determine whether the type of vocalization made a difference in the chimpanzees' shifting behavior, depending on the provisioned items (CBFCP versus NCEP, CBFCE versus NCEE). For Stage 2, we compared four combinations of the conditions to examine whether the vocalization type (CBFCAM versus NCEAM, CBFCPM versus NCEPM) or time of day (CBFCAM versus CBFCPM, NCEAM versus NCEPM) made a difference in the chimpanzees' shifting behavior (prediction (b)). Since we used the same dataset to test different combinations (i.e., two for Stage 1, four for Stage 2), we adjusted the significance level for these post hoc tests, using Bonferroni corrections, to $p \leq 0.05 / 2=0.025$ (Stage 1) and $p \leq 0.05 / 4=0.0125$ (Stage 2), respectively. We considered a probability of $0.025<p \leq 0.05$ (Stage 1) and $0.0125<p \leq 0.05$ (Stage 2) as a non-significant tendency for these post hoc comparisons. When they yielded significant results, we also reported the effect sizes.

Finally, since we observed four groups that reside in different enclosures at Chimp Haven, we also performed Kruskal-Wallis tests to control for group differences in an attempt to avoid pseudoreplication at the group level (Kuhar, 2006) for both stages. When the Kruskal-Wallis test produced significant results, we followed up on the findings by post hoc Mann-Whitney tests. As with other post hoc analyses, we set the significance level at $p=0.0083$, using a Bonferroni correction $(\mathrm{p} \leq 0.05 / \mathrm{six}$ combinations of four group comparisons), for each stage. A probability of $0.0083<p \leq 0.05$ was considered as a non-significant tendency.

\section{Results}

\section{Stage 1}

The chimpanzees' shifting behavior did not differ between the two vocalization types, when the effect of the provisioned items was removed (total CBFC versus total NCE) $(z=-1.04, p=0.301$ ), and thus prediction (a) was not supported for Stage 1 . However, there was a significant difference in shifting behavior when the four conditions were compared (Friedman ANOVA, $\chi^{2}(3)=8.56, p=0.036$ ) (Figure 1). Post hoc Wilcoxon matched-pairs signed-rank tests revealed a significant difference between CBFCE versus NCEE. When enrichment was provided, the proportion of shifts was significantly higher for CBFCE than for NCEE $(z=-2.38, n=22, p=0.017, r=-0.23$ (small to medium effect) (median proportion for both CBFCE and NCEE: 1.00; mean proportion difference: 0.03)). However, we found no significant difference in the shifting behavior depending on the vocalization type when produce was provided (CBFCP versus NCEP) $(z=-1.52, p=0.128)$.

The proportion of shifts was significantly affected by group differences for two conditions: CBFCP $(H=11.76, \mathrm{df}=3, p=0.008)$ and $\operatorname{NCEE}(H=15.07, \mathrm{df}=3, p=0.002)$. For the CBFCP condition, Ed's group was significantly more likely to shift to the outdoor enclosure than Kasey's group (Table 1). Similarly, for the NCEE condition, both Ed's and Emma's groups were significantly more likely to shift than Kasey's group. 


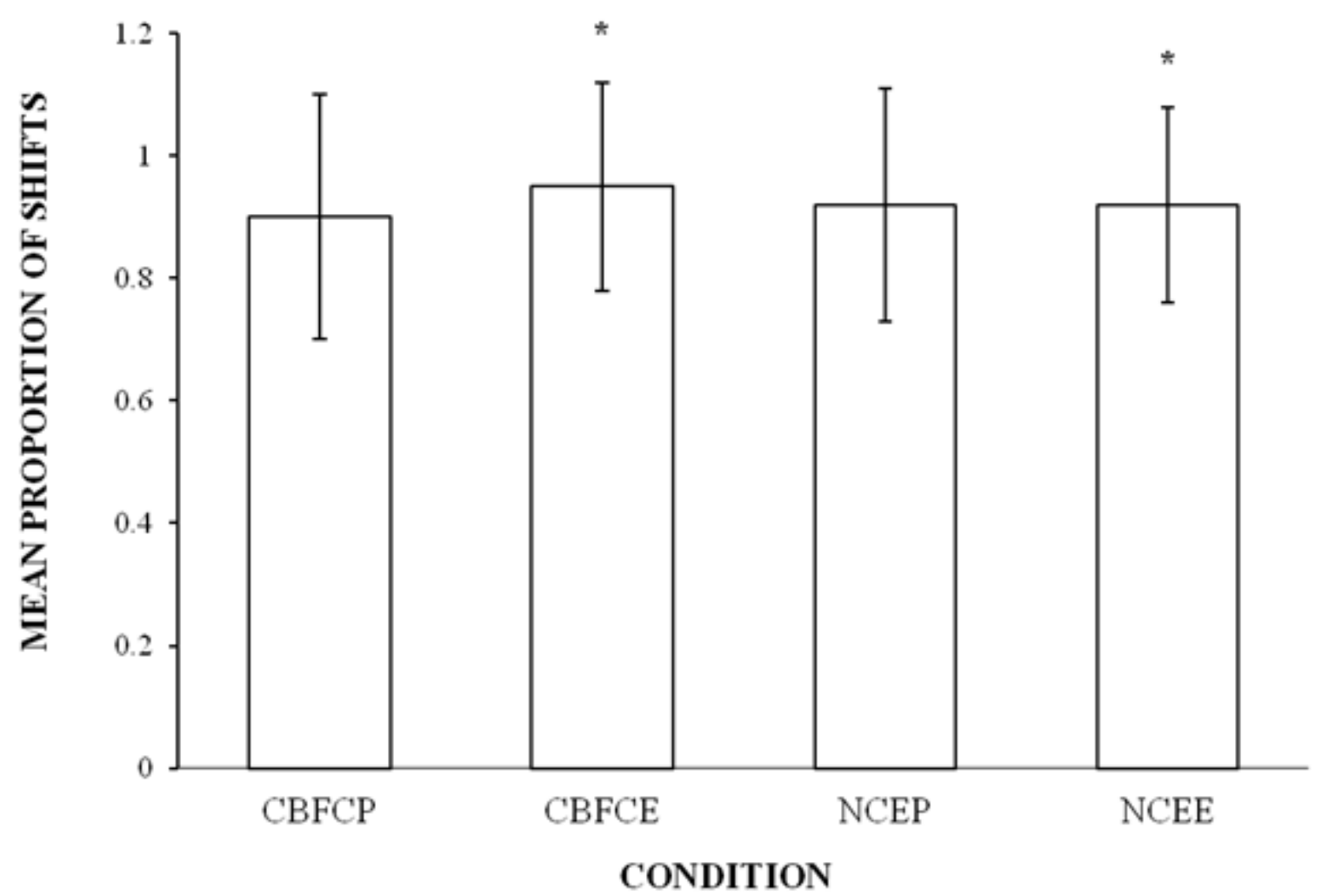

Figure 1. The mean proportion of shifts in each condition in Stage 1.

*There was a significant difference between chimpanzee based food calls enrichment (CBFCE) and name calling and encouragements enrichment (NCEE) during Stage $1, p=0.017$, Wilcoxon matched-pairs signed-rank test.

Table 1

Stage 1 Results of Mann-Whitney Tests: Group Comparisons that Produced Significant Results ${ }^{1,2}$

\begin{tabular}{llll}
\hline Condition: CBFCP & Level of Significance & Condition: NCEE & Level of Significance \\
\hline Conan vs. Kasey & $U=65, p=0.043(\mathrm{~ns}), r=-0.40$ & Ed vs. Conan & $U=74, p=0.016(\mathrm{~ns}), r=-0.43$ \\
Ed vs. Kasey & $U=36.5, p=0.002, r=-0.59$ & Ed vs. Kasey & $U=38, p=0.002, r=-0.39$ \\
Emma vs. Kasey & $U=36, p=0.028(\mathrm{~ns}), r=-0.45$ & Emma vs. Conan & $U=55, p=0.033(\mathrm{~ns}), r=-0.40$ \\
& & Emma vs. Kasey & $U=29, p=0.006, r=-0.56$
\end{tabular}

\footnotetext{
${ }^{1}$ This table includes group pairs that produced significant results or non-significant tendencies only.

${ }^{2}$ Individuals in the group in bold text were significantly more likely to shift to the outdoor enclosure than those in the group in plain text.
}

\section{Stage 2}

The chimpanzees' shifting behavior tended to differ between the total CBFCs versus the total NCE (Wilcoxon matched-pairs signed-rank test, $z=-1.68, n=38, p=0.093$ ); therefore, prediction (a) was not supported (Figure 2). Their shifting behavior significantly differed across the four conditions (Friedman ANOVA, $\chi^{2}(3)=11.98, p=0.0007$ ) (Figure 3). Although post hoc Wilcoxon matched-pairs signed-rank tests revealed a significant difference in shifting behavior between CBFCAM versus CBFCPM and between 
NCEAM versus NCEPM, prediction (b) was not supported; specifically, the proportion of shifts was significantly higher with CBFCAM than $\operatorname{CBFCPM}(z=-2.53, n=34, p=0.012, r=-0.26$ (small to medium effect) (median proportion for both conditions, 0.90; mean proportion difference, 0.12)). This was also the case for NCE ( $z=-2.71, n=39, p=0.007, r=-0.27$ (small to medium effect) (median proportion for both conditions: 0.90; mean proportion difference: 0.14$)$ ). Thus, for both types of vocalizations, the chimpanzees were significantly more likely to shift to the outdoor enclosure in the morning than in the afternoon. In fact, this was the case for the general tendency of shifting regardless of the vocalization type (Wilcoxon matchedpairs signed-rank test: $Z=-2.67, n=41, p=0.008, r=-0.27$ (small to medium effect)). As with Stage 1 , the proportion of shifts was significantly affected by group differences, but only for one condition, NCEPM $(H=9.78, \mathrm{df}=3, p=0.021)$; Mann-Whitney tests revealed that Ed's group was significantly more likely to shift to the outdoor enclosure than Kasey's group (Table 2).

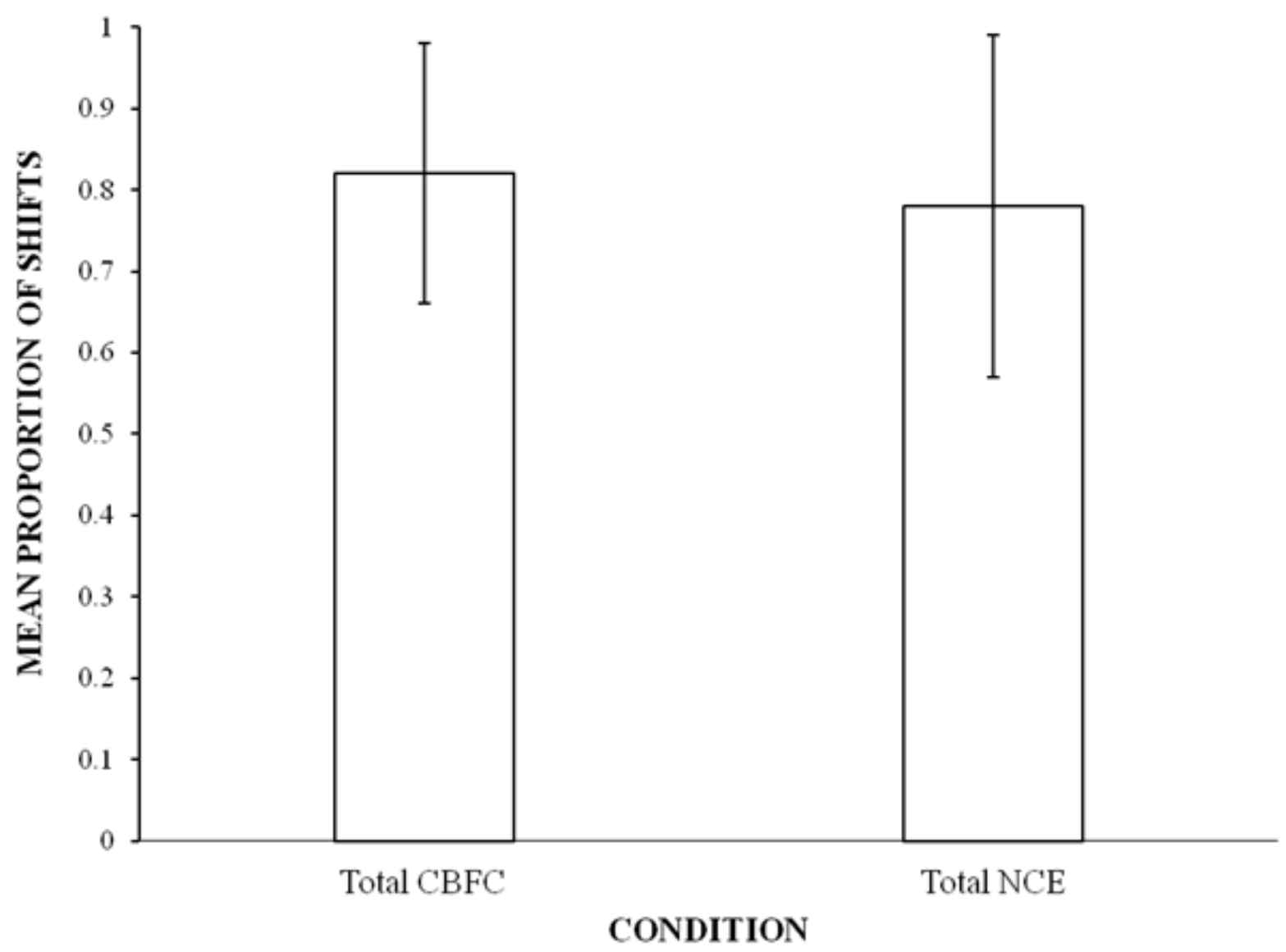

Figure 2. The mean proportion of overall shifts for Stage 2. There was a non-significant tendency between chimpanzee based food calls (CBFC) and total name calling and encouragements (NCE), $p=0.093$ (non-significant tendency), Wilcoxon matched-pairs signed-rank test. 


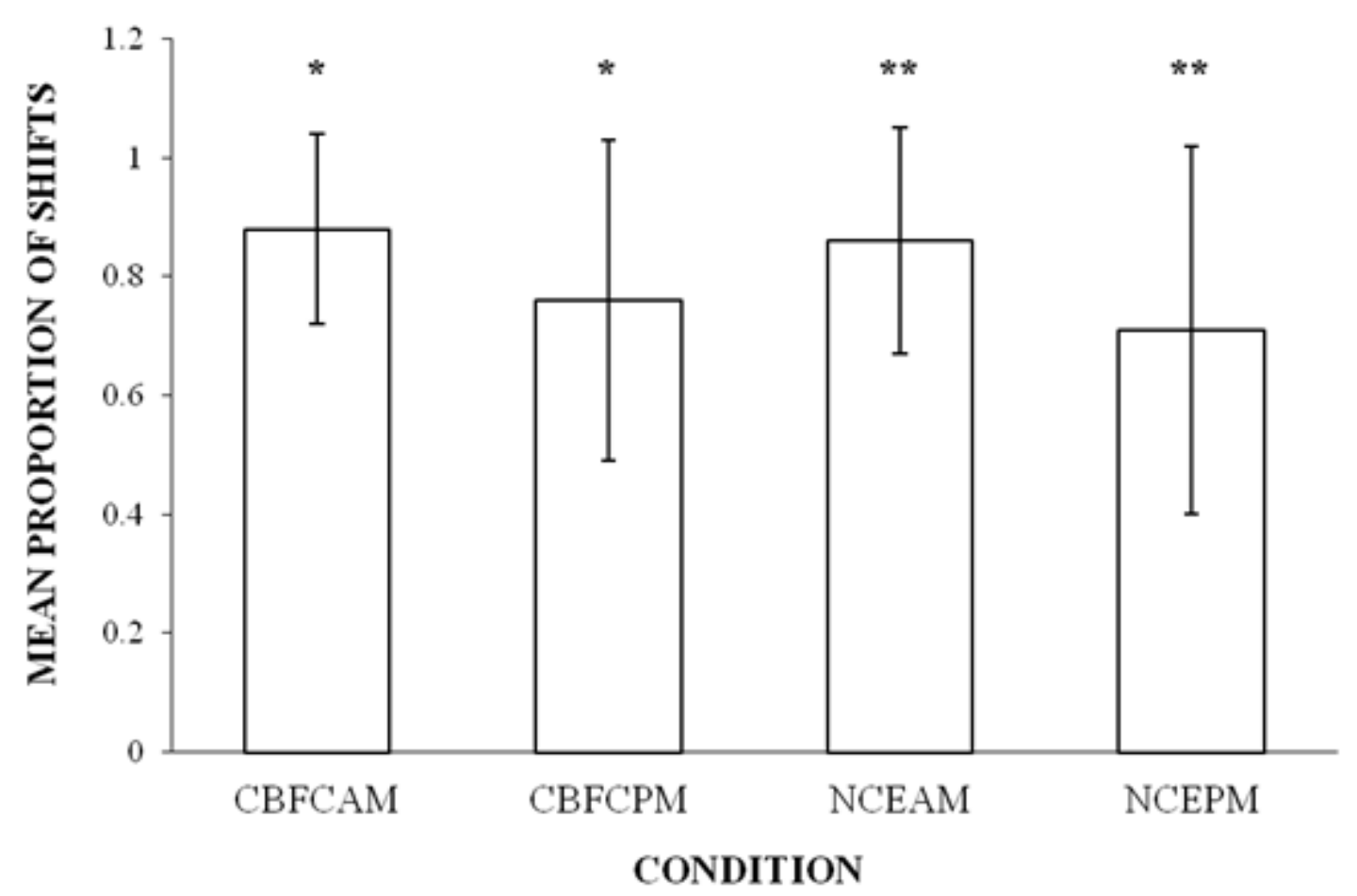

Figure 3. The mean proportion of shifts in each condition in Stage 2.

*There were significant differences between chimpanzee based food calls in the morning (CBFCAM) and chimpanzee based food calls in the afternoon (CBFCPM), $p=0.012$, Wilcoxon matched-pairs signed-rank test; and between name calling and encouragements in the morning (**NCEAM) and name calling and encouragements in the afternoon (NCEPM), $p=0.007$, Wilcoxon matched-pairs signed-rank test.

Table 2

Stage 2 Results of Mann-Whitney Tests: Group Comparisons that Produced Significant Results ${ }^{1,2}$

\begin{tabular}{|c|c|}
\hline Condition: NCEPM & Level of Significance \\
\hline Ed vs. Kasey & $U=37.5, p=0.005, r=-0.53$ \\
\hline Henry vs. Kasey & $U=25, p=0.014(\mathrm{~ns}), r=-0.51$ \\
\hline
\end{tabular}

\section{Discussion}

In this study, we examined whether utilizing more species-typical behavior in the form of CBFCs increased cooperation in shifting into outdoor enclosures. Regardless of the method of communication, the positive nature of the calling behavior by caregiver to chimpanzees was effective in encouraging chimpanzees to shift into different housing areas; however, time of day seemed to have a greater impact on the movement of chimpanzees. Chimpanzees were most likely to shift in the morning with either type of communication. For Stage 1, the chimpanzees shifted for both produce and enrichment for an average of $90 \%$ of the observations, regardless of the type of vocalization. Chimpanzees are highly food motivated and will announce the presence of high quality foods to their group (Goodall, 1986; Mitani, 1994). This increases the probability of the individuals approaching a food source (in this case, the caregiver or 
enrichment technician). When caregivers employed a CBFC, slightly more chimpanzees (95\%) shifted into the outdoor area than when caregivers simply called chimpanzees' names and encouraged them to come out for enrichment (92\%). At Chimp Haven, food barking is usually associated with food-based enrichment by the enrichment technician versus the usual NCE employed by most caregivers for produce and shifting for husbandry purposes. The CBFC may have adopted a more specific meaning, informing the chimpanzees that enrichment, not the normal daily produce, was on the way.

In Stage 1, the type of call only made a difference during the provision of enrichment and not with the provision of produce. This result may have been different with a larger sample size, providing caregivers with more training on how to mimic a CBFC and combining the food barking by caregivers with the provision of enrichment. Both types of calls, when combined with either type of food, promoted cooperative movement of the chimpanzees, which we believe fosters a positive HAR. This study shows that this type of relationship can assist in moving large or difficult species such as chimpanzees. This is similar to the research on positive reinforcement training and shifting chimpanzees, which allows for the chimpanzee to choose to receive a food item for behaving appropriately after receiving a verbal cue (Bloomsmith et al., 1998; Lambeth et al., 2006).

For Stage 2, in order to determine differences between CBFCs and NCE, we controlled for the type of food provisioned by restricting it to produce only, and we limited the natural variation in caregiver calls by having only an enrichment technician produce both types of call. For both types of call, in contrast to prediction (b), chimpanzees were more likely to shift to the outdoor areas in the morning (CBFC, $88 \%$; NCE, $86 \%)$ versus the afternoon $(76 \%, 71 \%$, respectively). This result contradicts the general hypothesis that human presence is stressful for the animals (Alford et al., 1992), which is typically in the morning. This also differs from Ross et al. (2010) in which the chimpanzees showed increased stress when being moved to a different area for husbandry and cleaning purposes. Comparatively, our study involves shifting animals to a larger outdoor area, only limiting their access to the smaller indoor area briefly, rather than moving them from a larger outdoor/exhibit to a smaller indoor area.

It is possible that a level of predictable signaling on the part of the caregivers to the chimpanzees is occurring during the morning. This signaling may allow the chimpanzees to avoid a stressful event (e.g., shutting doors for cleaning) or participate in more pleasant events (e.g., receiving fruits and vegetables) (Bassett \& Buchanan-Smith, 2007). Thus, the morning may provide more opportunities for positive interactions between caregivers and chimpanzees. These interactions are necessary to facilitate shifting behavior that typically occurs in the morning. Indeed, studies such as Jensvold (2008), Chelluri et al. (2013) and Bloomsmith et al. (1998) have shown that fostering these positive HARs can actually increase chimpanzee cooperation and decrease the agonistic encounters between chimpanzees and caregivers.

Anticipating events that are pleasurable or avoiding events that are stressful can impact behavior in similar ways, especially with the use of positive reinforcement training (Bassett \& Buchanan-Smith, 2007; Bloomsmith et al., 1998; Pomerantz \& Terkel, 2009). Many husbandry schedules necessitate cleaning early in the day, requiring animals to shift, which might be stressful (Bassett et al., 2003; Saibaba et al., 1996). Chimpanzees at Chimp Haven are shifted daily in the morning to outdoor enclosures for cleaning inside bedrooms, they are not regularly shifted in the afternoon. Being able to predict events such as shifting for cleaning can be desirable as it allows the animals some control of their environment (Bassett \& Buchanan-Smith, 2007). Chimpanzees at Chimp Haven may prefer to shift in the mornings because they are accustomed to this routine despite the increase in potentially stressful husbandry tasks. In contrast, Bloomsmith and Lambeth (1995) found that during unpredictable feeding schedules, chimpanzees were more active, coprophagy levels decreased, and welfare generally increased. However, this may be due to the shorter 150-min period during which the unpredictable feeding occurred, while our study examined differences between morning and afternoon over a longer time period. There is little in the literature regarding changes in chimpanzee behavior that are related to these larger differences in time of day when husbandry activities (other than feeding) are occurring, suggesting that this may be an important area of future study.

In this study we found that some groups shifted more reliably than other groups. We suggest that this may be due to differences between the home enclosures of the different groups. If a group was in a 
corral for example, they merely have to step outside from their indoor area to shift. In contrast, a group in one of the yards needs to travel through an overhead chute to reach the outdoor enclosure, necessitating the expenditure of additional energy. Other possible explanations for these group differences studies might include the age, health status, background, or rearing history of the chimpanzees, which could be explored in future studies.

Understanding of typical species behavior, positive reinforcement training, and the encouragement of strong HARs all work together to increase overall animal welfare (Claxton, 2011). In this study, we attempted to use species-typical behavior (CBFCs and NCE) for shifting purposes to build a more positive relationship between the chimpanzees and their caregivers. Our results partially support the hypothesis that a species-typical vocalization (CBFCs) helped the chimpanzees shift slightly more effectively, but only when it was combined with the provisioning of enrichment items. At the same time, the difference between this vocalization and NCE does not appear to affect the chimpanzees' shifting behavior when produce is provided; leading us to conclude that positive communication between caregivers and the chimpanzees in general can be an effective tool, but that a predictable routine or predictable signaling may be more important. Our results show that time of day affected the shifting behavior of sanctuary chimpanzees; the chimpanzees were more likely to shift in the morning than in the afternoon, possibly because it has become a predictable event, which was signaled by caregiver presence, in their daily routine. Our findings are consistent with these interpretations; however, additional information regarding the chimpanzees' behavior or physiological state would be helpful to fully determine whether these interactions had positive effects on the chimpanzees' welfare. For future research, we further suggest investigating other factors that may influence the shifting of chimpanzees for husbandry tasks, such as rank, rearing history, and individual preferences. In conclusion, this study suggests that both species-typical communication and positive interactions in the form of name-calling and encouragement can be effective tools to increase the cooperation of chimpanzees during regular husbandry procedures at a sanctuary.

\section{Acknowledgments}

The authors would like to acknowledge the Chimp Haven team and in particular all of the caregivers who participated in Stage 1 of this study. We would also like to thank Steve Snodgrass for his editing skills. Chimp Haven is an AAALAC International-accredited facility, and abides by the Animal Welfare Act and Standards of Care for Chimpanzees Held in the Federally Supported Chimpanzee Sanctuary System (Federal Register, 2008). The views and opinions expressed in this publication represent the authors' views alone, and do not express or imply the views, endorsement, or financial support of the federal government or any of its agencies, including the National Institutes of Health, unless otherwise stated by an authorized representative thereof.

\section{References}

Alford, P. L., Nash, L. T., Fritz, J., \& Bowen, J. A. (1992). Effects of management practices on the timing of captive chimpanzee births. Zoo Biology, 11, 253-260. http://doi.org/10.1002/zoo.1430110405

Aureli, F., \& de Waal, F. B. M. (1997). Inhibition of social behavior in chimpanzees under high-density conditions. American Journal of Primatology 41, 213-228. http://doi.org/10.1002/(SICI)10982345(1997)41:3<213::AID-AJP4>3.0.CO;2-\#

Bassett, L., \& Buchanan-Smith, H. M. (2007). Effects of predictability on the welfare of captive animals. Applied Animal Behaviour Science, 102, 223-245. http://doi.org/10.1016/j.applanim.2006.05.029

Bassett, L., Buchanan-Smith, H. M., McKinley, J., \& Smith, T. E. (2003). Effects of training on stress-related behavior of the common marmoset (Callithrix jacchus) in relation to coping with routine husbandry procedures. Journal of Applied Animal Welfare Science, 6, 221-233. doi:10.1207/S15327604JAWS0603_07

Bayne, K. (2002). Development of the human-research animal bond and its impact on animal well-being. ILAR Journal, 43, 4-9. http://doi.org/10.1093/ilar.43.1.4 
Bayne, K. A., Dexter, S. L., \& Strange, G. M. (1993). The effects of food treat provisioning and human interaction on the behavioral well-being of rhesus monkeys (Macaca mulatta). Contemporary Topics in Laboratory Animal Science / American Association for Laboratory Animal Science, 33, 6-9.

Bloomsmith, M. A., \& Lambeth, S. P. (1995). Effects of predictable versus unpredictable feeding schedules on chimpanzee behavior. Applied Animal Behaviour Science, 44, 65-74. http://doi.org/10.1016/01681591(95)00570-I

Bloomsmith, M. A., Stone, A. M., \& Laule, G. E. (1998). Positive reinforcement training to enhance the voluntary movement of group-housed chimpanzees within their enclosures. Zoo Biology, 17, 333-341. http://doi.org/10.1002/(SICI)1098-2361(1998)17:4<333::AID-ZOO6>3.0.CO;2-A

Brent, L. (2007). Life-long well-being: Applying animal welfare science to nonhuman primates in sanctuaries. Journal of Applied Animal Welfare Science, 10, 55-61. http://doi.org/10.1080/10888700701277626

Brosnan, S. F., \& de Waal, F. B. M. (2014). Evolution of responses to (un)fairness. Science, 346, 1251776-1251776. http://doi.org/10.1126/science.1251776

Carlstead, K. (2009). A comparative approach to the study of keeper-animal relationships in the zoo. Zoo Biology, 28, 589-608. http://doi.org/10.1002/zoo.20289

Chelluri, G. I., Ross, S. R., \& Wagner, K. E. (2013). Behavioral correlates and welfare implications of informal interactions between caretakers and zoo-housed chimpanzees and gorillas. Applied Animal Behaviour Science, 147, 306-315. http://doi.org/10.1016/j.applanim.2012.06.008

Claxton, A. M. (2011). The potential of the human-animal relationship as an environmental enrichment for the welfare of zoo-housed animals. Applied Animal Behaviour Science, 133, 1-10. http://doi.org/10.1016/j.applanim.2011.03.002

Coe, J. C., Fulk, R., \& Brent, L. (2001). Facility design. In L. Brent. (Ed.), The care and management of captive chimpanzees (pp. 39 - 81). San Antonio, TX: The American Society of Primatologists.

Dawkins, M. S. (1998). Evolution and Animal Welfare. The Quarterly Review of Biology, 73(3), 305-328.

Dawkins, M. S. (2003). Behaviour as a tool in the assessment of animal welfare. Zoology, 106(4), 383-387. http://doi.org/10.1078/0944-2006-00122

Dorsey, C. (2010). Chimpanzee (Pan troglodytes) care manual. Association of Zoos and Aquarium. AZA Ape TAG. Chimpanzee (Pan troglodytes) Care Manual. Association of Zoos and Aquariums, Silver Spring, MD.

Fultz, A., Brent, L., Breaux, S. D., \& Grand, A. P. (2013). An evaluation of nest-building behavior by sanctuary chimpanzees with access to forested habitats. Folia Primatologica, 84, 405-420. http://doi.org/10.1159/000353900

Gibson, J. M., Scavelli, S. A., Udell, C. J., \& Udell, M. A. R. (2014). Domestic dogs (Canis lupus familiaris) are sensitive to the "human" qualities of vocal commands. Animal Behavior and Cognition, 1, 281. http://doi.org/10.12966/abc.08.05.2014

Goodall, J. (1986). The chimpanzees of Gombe: Patterns of behavior. Cambridge, MA: Belknap Press.

Hauser, M. D., \& Wrangham, R. W. (1987). Manipulation of food calls in captive chimpanzees. A preliminary report. Folia Primatologica; International Journal of Primatology, 48, 207-10. http://doi.org/10.1159/000156298

Hemsworth, P. (2003). Human-animal interactions in livestock production. Applied Animal Behaviour Science, 81, 185-198. http://dx.doi.org/10.1016/S0168-1591(02)00280-0

Hemsworth, P. (2007). Ethical stockmanship. Australian Veterinary Journal, 85, 194-200. http://doi.org/10.1111/j.1751-0813.2007.00112.x

Hopkins, W. D., Taglialatela, J. P., \& Leavens, D. A. (2007). Chimpanzees differentially produce novel vocalizations to capture the attention of a human. Animal Behaviour, 73, 281-286. http://doi.org/10.1016/j.anbehav.2006.08.004

Hosey, G. (2008). A preliminary model of human-animal relationships in the zoo. Applied Animal Behaviour Science, 109(2-4), 105-127. http://doi.org/10.1016/j.applanim.2007.04.013

Hosey, G., \& Melfi, V. (2012). Human-animal bonds between zoo professionals and the animals in their care. Zoo Biology, 31, 13-26.

Hostetter, A. B., Cantero, M., \& Hopkins, W. D. (2001). Differential use of vocal and gestural communication by chimpanzees (Pan troglodytes) in response to the attentional status of a human (Homo sapiens). Journal of Comparative Psychology, 115, 337.

Itakura, S., Agnetta, B., Hare, B., \& Tomasello, M. (1999). Chimpanzee use of human and conspecific social cues to locate hidden food. Developmental Science, 2(4), 448-456.

Jensvold, M. L. A. (2008). Chimpanzee (Pan troglodytes) responses to caregiver use of chimpanzee behaviors. Zoo Biology, 27, 345-359. 
Kuhar, C. W. (2006). In the deep end: Pooling data and other statistical challenges of zoo and aquarium research. Zoo Biology, 25, 339-352.

Kessel-Davenport, A., \& Gutierrez, T. (1994). Training captive chimpanzees for movement in a transfer box. The Newsletter, Primate Foundation of Arizona, 6, 1-2.

Lambeth, S. P., Hau, J., Perlman, J. E., Martino, M., \& Schapiro, S. J. (2006). Positive reinforcement training affects hematologic and serum chemistry values in captive chimpanzees (Pan troglodytes). American Journal of Primatology, 68, 245-256. http://doi.org/10.1002/ajp.20148

Lambeth, S. P., Bloomsmith, M. A., \& Alford, P. L. (1997). Effects of human activity on chimpanzee wounding. Zoo Biology, 16(4), 327-333. http://doi.org/10.1002/(SICI)1098-2361(1997)16:4<327::AIDZOO4>3.0.CO;2-C

Mitani, J. C. (1994). Ethological studies of chimpanzee vocal behavior. In: R. W. Wrangham, W. C. McGrew, F. B. M. de Waal, P. Heltne and J. Goodall (Eds.), Chimpanzee Cultures (195-210). Chicago, IL: Chicago Academy of Sciences.

Nieuwenhuijsen, K., \& de Waal, F. B. M. (1982). Effects of spatial crowding on social behavior in a chimpanzee colony. Zoo Biology, 1, 5-28. http://doi.org/10.1002/zoo.1430010103

Perlman, J. E., Bloomsmith, M. A., Whittaker, M. A., McMillan, J. L., Minier, D. E., \& McCowan, B. (2012). Implementing positive reinforcement animal training programs at primate laboratories. Applied Animal Behaviour Science, 137, 114-126. http://doi.org/10.1016/j.applanim.2011.11.003

Pomerantz, O., \& Terkel, J. (2009). Effects of positive reinforcement training techniques on the psychological welfare of zoo-housed chimpanzees (Pan troglodytes). American Journal of Primatology, 71, 687-695. http://doi.org/10.1002/ajp.20703

Reinhardt, V. (2004). Common husbandry-related variables in biomedical research with animals. Laboratory Animals, 38, 213-235. http://doi.org/10.1258/002367704323133600

Ross, S. R., Wagner, K. E., Schapiro, S. J., \& Hau, J. (2010) Ape behavior in two alternating environments: Comparing exhibit and short-term holding areas. American Journal of Primatology, 72, 951-959. http://doi.org/10.1002/ajp.20857

Saibaba, P., Sales, G. D., Stodulski, G., \& Hau, J. (1996). Behaviour of rats in their home cages: Daytime variations and effects of routine husbandry procedures analysed by time sampling techniques. Laboratory Animals, 30, 13-21. http://doi.org/10.1258/002367796780744875

Slocombe, K. E., Kaller, T., Turman, L., Townsend, S. W., Papworth, S., Squibbs, P., \& Zuberbühler, K. (2010). Production of food-associated calls in wild male chimpanzees is dependent on the composition of the audience. Behavioral Ecology and Sociobiology, 64, 1959-1966. http://doi.org/10.1007/s00265-010-1006-0

Slocombe, K. E., \& Zuberbühler, K. (2006). Food-associated calls in chimpanzees: Responses to food types or food preferences? Animal Behaviour, 72, 989-999. http://doi.org/10.1016/j.anbehav.2006.01.030

Waitt, C., Buchanan-Smith, H. M., \& Morris, K. (2002). The effects of caretaker-primate relationships on primates in the laboratory. Journal of Applied Animal Welfare Science, 5, 309-319. http://doi.org/10.1207/S15327604JAWS0504_05 